\title{
Erratum zu: Digital Leadership, Agile Change und die Emotion Economy
}

\section{Kapitel 2 in M. Kupiek, Digital Leadership, Agile Change und die Emotion Economy, https://doi.org/10.1007/978-3-658-31042-4_2}

Das Wort „geändert“ in der Tabelle auf S. 49 wurde entfernt.

\section{Kapitel 5 in M. Kupiek, Digital Leadership, Agile Change und die Emotion Economy, https://doi.org/10.1007/978-3-658-31042-4_5}

Der auf S. 134 fälschlicherweise gesetzte Kommentar des Autors „JETZT HIER DAS CARTOON EInFÜGEN. DANACH DER FOLGENDE SATZ“ wurde entfernt.

Die aktualisierte Version dieses Kapitels finden Sie unter https://doi.org/10.1007/978-3-658-31042-4_2 https://doi.org/10.1007/978-3-658-31042-4_5 